\title{
Do Words Matter? Stigmatizing Language and the Transmission of Bias in the Medical Record
}

\author{
Anna P. Goddu, MSC' , Katie J. O'Conor, BA' ', Sophie Lanzkron, MD, MHS', \\ Mustapha O. Saheed, MD', Somnath Saha, MD, MPH ${ }^{4,5}$, Monica E. Peek, MD, MPH, MSc 6 , \\ Carlton Haywood, Jr., PhD, MA', and Mary Catherine Beach, MD, MPH
}

\begin{abstract}
'Johns Hopkins University School of Medicine, Baltimore, MD, USA; ${ }^{2}$ Division of Hematology, Johns Hopkins University School of Medicine, Baltimore, MD, USA; ${ }^{3}$ Department of Emergency Medicine, Johns Hopkins University School of Medicine, Baltimore, MD, USA; ${ }^{4}$ Section of General Internal Medicine, VA Portland Health Care System, Portland, OR, USA; ${ }^{5}$ Division of General Internal Medicine and Geriatrics, Oregon Health and Science University, Portland, OR, USA; 'Section of General Internal Medicine, The University of Chicago, Chicago, IL, USA.
\end{abstract}

BACKGROUND: Clinician bias contributes to healthcare disparities, and the language used to describe a patient may reflect that bias. Although medical records are an integral method of communicating about patients, no studies have evaluated patient records as a means of transmitting bias from one clinician to another.

OBJECTIVE: To assess whether stigmatizing language written in a patient medical record is associated with a subsequent physician-in-training's attitudes towards the patient and clinical decision-making.

DESIGN: Randomized vignette study of two chart notes employing stigmatizing versus neutral language to describe the same hypothetical patient, a 28-year-old man with sickle cell disease.

PARTICIPANTS: A total of 413 physicians-in-training: medical students and residents in internal and emergency medicine programs at an urban academic medical center (54\% response rate).

MAIN MEASURES: Attitudes towards the hypothetical patient using the previously validated Positive Attitudes towards Sickle Cell Patients Scale (range 7-35) and pain management decisions (residents only) using two multiple-choice questions (composite range 2-7 representing intensity of pain treatment).

KEY RESULTS: Exposure to the stigmatizing language note was associated with more negative attitudes towards the patient (20.6 stigmatizing vs. 25.6 neutral, $p<0.001$ ). Furthermore, reading the stigmatizing language note was associated with less aggressive management of the patient's pain (5.56 stigmatizing vs. 6.22 neutral, $p=0.003$ ). CONCLUSIONS: Stigmatizing language used in medical records to describe patients can influence subsequent physicians-in-training in terms of their attitudes towards the patient and their medication prescribing behavior. This is an important and overlooked pathway by which bias can be propagated from one clinician to another. Attention to the language used in medical records may help to promote patient-centered care and to reduce healthcare disparities for stigmatized populations.

Received July 9, 2017

Revised November 13, 2017

Accepted December 13, 2017

Published online January 26, 2018
KEY WORDS: bias; stigma; language; disparities; medical record; communication; clinical decision-making.

$\mathrm{J}$ Gen Intern Med 33(5):685-91

DOI: $10.1007 / \mathrm{s} 11606-017-4289-2$

(c) Society of General Internal Medicine 2018

\section{INTRODUCTION}

It is well documented that patients are not treated equally in our healthcare system: some receive poorer quality of healthcare than others based on their racial/ethnic identity, ${ }^{1-4}$ independent of social class. Others, such as older adults ${ }^{5,6}$ and individuals with low health literacy, ${ }^{7,8}$ obesity, ${ }^{9,10}$ and substance use disorders ${ }^{11}$ may also be viewed negatively by health professionals in a way that adversely impacts their healthcare quality. Implicit bias among clinicians is one factor that perpetuates these disparities. ${ }^{1,12,13}$ Implicit bias is the automatic activation of stereotypes derived from common cultural experiences, which may override deliberate thought and influence one's judgment in unintentional and unrecognized ways, ${ }^{2,14,15}$ and may affect communication behaviors and treatment decisions. ${ }^{3,16-18}$

Clinicians may acquire implicit bias towards patients from one another when communicating verbally or when writing or reading medical records; physicians-in-training may absorb these attitudes as part of the "hidden curriculum" of medical training. ${ }^{19-22}$ Few studies have examined the medical record as a mechanism for transmitting bias from one clinician to another. A recent randomized study demonstrated that physicians who read a vignette with the term "substance abuser" as opposed to "having a substance use disorder" were more likely to agree that the depicted character was personally culpable and should have punitive measures taken against him or her. ${ }^{11}$ Those participants were also less likely to agree with the notion that a "substance abuser" needed treatment as compared to a person "with substance use disorder." 23

In this study, we explored whether stigmatizing language written in a patient medical record was associated with a subsequent physician-in-training's attitudes towards the patient and clinical decision-making. We hypothesized that 
resident physicians and medical students who read a chart note using stigmatizing language to describe a patient would have more negative attitudes about the patient and would treat the patient's pain less aggressively than those who read a chart note using neutral language.

\section{METHODS}

We employed an experimental vignette study design in which residents and medical students were randomized to read one of two chart notes presenting medically identical information about a hypothetical patient with sickle cell disease (SCD). The first chart note utilized stigmatizing language and details; the second used neutral language. After reading the chart note, participants completed a survey assessing their attitudes regarding the patient (residents and students) and treatment decisions (residents only) for the patient. We focused on a patient with SCD, as there is significant evidence that these patients are stigmatized by clinicians and experience inadequate pain management, conflicts with staff, and lack of respect. ${ }^{24}$ We decided to focus on medical students and residents, as training is a time of socialization through the "hidden curriculum" $" 19$ and thus a potential point of intervention. We selected residents in emergency medicine and internal medicine because they care for patients with SCD presenting with pain crises. Hypothetical chart notes were used because vignettes are a robust and efficient method to systematically assess variation in opinion and decision-making, without confounding by other patient characteristics. ${ }^{25,26}$ All research activities were approved by the academic medical center's institutional review board.

\section{Study Setting and Sample}

The study was performed at a large, urban academic medical center. Residents in the medical center's two internal medicine (IM) programs and one emergency medicine (EM) program were recruited via emails cosigned by their program directors. Medical students were recruited with an email from a student on the study team, cosigned by the principal investigator. The survey was administered online via Qualtrics, which randomized participants to the stigmatizing language or neutral language arm of the study. As incentive for their participation, residents were given $\$ 25$ gift cards. Medical students were entered into a lottery for one of five $\$ 100$ gift cards. Two reminders were sent via email at 10-day intervals.

\section{Survey Vignettes}

Two vignettes were created by the study team, written in the format of chart notes. The vignette describes Mr. R, a hypothetical 28-year-old man with SCD who presents to the emergency department (ED) with a vaso-occlusive crisis. In Section 1 of the vignette, the admitting physician describes Mr. R's chief complaint, history of present illness, and
Table 1 Text Employed in the Vignettes

\begin{tabular}{l}
\hline \hline Neutral language chart note \\
\hline Section 1 \\
Mr. R is a 28 -year old man with \\
sickle cell disease and chronic left \\
hip osteomyelitis who comes to \\
the ED with $10 / 10$ pain in his \\
arms and legs. He has about $8-10$ \\
pain crises per year, for which he \\
typically requires opioid pain \\
medication in the ED. At home, \\
he takes 100 mg OxyContin BID \\
and oxycodone 5 mg for break- \\
through pain. Over the past few \\
days, he has taken 2 tabs every $4-$ \\
6 hours. About 3 months ago, he \\
moved to a new apartment and \\
now has to wheel himself in a \\
manual wheelchair up 3 blocks \\
from the bus stop.
\end{tabular}

He spent yesterday afternoon with friends and wheeled himself around more than usual, which caused dehydration due to the heat. He believes that this, along with recent stress, precipitated his current crisis. The pain is aching in quality, severe (10/10), and not alleviated by his home pain medication regimen.

On physical exam, he is in obvious distress. He has no fever and his pulse ox is $96 \%$ on RA. The rest of the physical exam is normal other than tenderness to palpation on the left hip.

\section{Stigmatizing language chart} note

Mr. $\mathrm{R}$ is a 28-year old sickle cell patient with chronic left hip osteomyelitis who comes to the ED stating he has 10/10 pain "all up in my arms and legs." He is narcotic dependent and in our ED frequently. At home he reportedly takes $100 \mathrm{mg}$ OxyContin BID and oxycodone $5 \mathrm{mg}$ for breakthrough pain. Over the past few days, he says that he has taken 2 tabs every 4-6 hours. About 3 months ago, patient states that the housing authority moved him to a new neighborhood and he now has to wheel himself in a manual wheelchair up 3 blocks from the bus stop.

Yesterday afternoon, he was hanging out with friends outside McDonald's where he wheeled himself around more than usual and got dehydrated due to the heat. He believes that this, along with some "stressful situations," has precipitated his current crisis. Pain is aching in quality, severe $(10 / 10)$, and has not been helped by any of the narcotic medications he says he has already taken.

On physical exam, he appears to be in distress. He has no fever and his pulse ox is $96 \%$ on RA. The rest of the physical exam is normal although he reports tenderness to palpation on the left hip.

Section 2

After 1 hour, the nurse documents:

Mr. R is sleeping but easily arousable and seems distressed. $\mathrm{He}$ is not tolerating the oxygen mask and still has $10 / 10$ pain. His girlfriend is by his side but will need to go home soon.

Mr. $\mathrm{R}$ is sleeping but easily arousable and has been cussing at nurse. He refuses to wear his oxygen mask and is insisting that his pain is "still a 10." His girlfriend is lying on the bed with shoes on and requests a bus token to go home.

physical exam findings. In Section 2, a nurse documents information about Mr. R from an hour later. The chart notes are shown in Table 1.

The two chart notes differed in the use of stigmatizing versus neutral language. The stigmatizing chart note was a written composite based on text abstracted from medical records from the same medical center. ${ }^{27}$ We defined stigma after Bruce Link and Jo Phelan, who conceptualize stigma as elements of labeling, stereotyping, separating, status loss, and discrimination co-occurring in a power situation that allows these processes to unfold. ${ }^{28}$ Stigmatizing language in patient charts was characterized by three linguistic features, as elaborated in prior work ${ }^{27}$ : casting doubt on the patient's pain (e.g. 
insisting that his pain is "still a 10" vs. still has $10 / 10$ pain), portraying the patient negatively (with irrelevant or unnecessary indicators of lower socioeconomic status such as hanging out with friends outside McDonald's), and implying patient responsibility with references to uncooperativeness (e.g. he refuses his oxygen mask vs. he is not tolerating the oxygen mask). In addition, we included linguistic variations such as using the term "narcotic" in the stigmatizing chart note vs. "opioid" in the neutral note. The neutral chart note was written by the study team to serve as a comparison against the stigmatizing language note, while remaining realistic.

The survey was reviewed, piloted by members of the study team and ten medical students, and revised based on feedback. Pilot data responses were not included in the final data set.

\section{Primary Outcomes}

Pain Management Intensity. After reading Section 1, residents were asked to choose among four options for pain medication dosing (ketorolac $60 \mathrm{mg} \mathrm{IM}$; morphine $4 \mathrm{mg} \mathrm{IV}$; hydromorphone $1 \mathrm{mg}$ IV; hydromorphone $4 \mathrm{mg} \mathrm{IV}$ ). They were then presented with Section 2 and asked how they would adjust their management plan (change to oral pain medication and discharge; give second dose of IV medication; increase dose of IV medication; tell patient it is too early for additional opioids and offer ketorolac). The two medication management questions were combined (and the options for morphine $4 \mathrm{mg}$ IV and hydromorphone $1 \mathrm{mg}$ IV collapsed based on clinical equivalence) to yield a score from 2 to 7 , with higher scores indicating more aggressive pain management. Medical students read both sections of the chart note at the same time and did not answer the pain management questions.

Attitudes towards the Patient. All study participants completed an abbreviated Positive Attitudes towards Sickle Cell Patients Scale (PASS). ${ }^{24}$ Seven of the 10 items of the PASS were utilized to directly assess attitudes towards the hypothetical patient, using a five-point Likert scale for a total score range of 7-35, with higher scores representing more positive attitudes. Because the PASS was developed as a scale to measure attitudes towards actual patients rather than hypothetical patients, we did not view the remaining three items (which ask the respondent to indicate how much they respect, empathize, and like the patient) as adaptable to the vignette scenario.

\section{Secondary Outcome}

Perceptions of Vignette Physician Attitudes. To assess whether participants perceived bias within the note, the other three PASS items were modified to ask the participant to rate how much the admitting physician liked, respected, and empathized with the patient described (vs. participants rating their own attitudes towards the patient). These three questions were combined into a score ranging from 3 to 15 , with higher scores indicating that the respondent perceived more positive attitudes by the admitting physician towards the patient. After these questions, and without the possibility of returning to change their answer, an additional, open-ended question asked the respondent to indicate what features of the note might convey positive or negative attitudes towards the patient.

\section{Other Predictor Variables}

Comfort in Dosing Pain Medication. The residents rated their comfort in dosing pain medication on a scale from 1 to 10 , with a higher score representing greater comfort.

Demographics. We collected information from respondents about their year of training (in medical school or residency), residency type (IM vs. EM), gender, race, and ethnicity.

\section{Statistical Analysis}

We used chi-square analyses to compare characteristics of respondents between the two study arms. After noting no significant differences, we used $t$ tests (for pain management intensity, normally distributed) and Wilcoxon rank-sum tests (for attitudes towards patients, not normally distributed) to calculate differences between arms.

We conducted several secondary analyses. We performed $t$ tests to assess potential differences between students and residents, between black and non-black respondents, and between EM and IM residents in attitudes towards the patient. To evaluate whether residents who were more comfortable dosing pain medication chose more aggressive pain management, we performed a linear regression between the medication score and comfort score among all residents and among residents within each study arm. Finally, we explored respondents' perceptions of bias on the part of the (hypothetical) admitting physician using a Wilcoxon rank-sum test to calculate the difference in the three-item attitude score- how much respondents perceived that the admitting physician liked and had respect and empathy for the patient — between the stigmatizing and neutral language chart notes. We explored and described the reasons given in the open-ended comments.

\section{RESULTS}

\section{Study Sample}

Of the 413 study participants (54\% response rate), $42.8 \%$ were female, $43.5 \%$ were residents, and $14 \%$ were Hispanic/Latino. Most respondents were white (54.7\%), 26.9\% were Asian, and $10.4 \%$ identified as black or African American. Respondent 
characteristics including year of training, ethnicity, gender, and race were balanced between study arms (Table 2).

\section{Impact of the Stigmatizing Language Chart Note on Attitudes and Pain Management}

Differences in attitudes and pain management intensity between arms are shown in Figure 1a and b. Exposure to the stigmatizing language chart note negatively influenced resident and medical student attitudes towards the hypothetical patient as measured by the PASS scores: all respondents (20.3 stigmatizing language vs. 25.1 neutral language, $p<0.001$; Fig. 1a). This was true of medical students (21.6 stigmatizing vs. 26.6 neutral, $p<0.001)$ and residents (18.7 stigmatizing vs. 23.0 neutral, $p<0.001)$.

Resident physicians who read the stigmatizing language chart note prescribed pain medication less aggressively than those who read the neutral language chart note (4.7 vs. 5.3, $p<0.001$; Fig. 1b).

Table 2 Characteristics of Survey Respondents

\begin{tabular}{|c|c|c|c|c|}
\hline No. (\%) & Overall & $\begin{array}{l}\text { Neutral } \\
\text { chart note }\end{array}$ & $\begin{array}{l}\text { Stigmatizing } \\
\text { chart note }\end{array}$ & $\begin{array}{l}p \\
\text { value }\end{array}$ \\
\hline Total no. & $\begin{array}{l}413 \\
(100)\end{array}$ & $208(50.3)$ & 205 (49.6) & \\
\hline \multicolumn{5}{|l|}{ Demographics } \\
\hline Female & $\begin{array}{l}177 \\
(42.8)\end{array}$ & $89(42.7)$ & $88(42.9)$ & 0.85 \\
\hline \multicolumn{5}{|c|}{ Self-reported race } \\
\hline Asian & $\begin{array}{l}111 \\
(26.9)\end{array}$ & $60(28.9)$ & $51(24.9)$ & 0.36 \\
\hline Black/AA & $\begin{array}{l}43 \\
(10.4)\end{array}$ & $21(10.1)$ & $22(10.7)$ & 0.83 \\
\hline White & $\begin{array}{l}226 \\
(54.7)\end{array}$ & $115(55.3)$ & $111(54.2)$ & 0.81 \\
\hline Other race & $27(6.5)$ & $12(5.8)$ & $15(7.32)$ & 0.53 \\
\hline Hispanic/Latino & $\begin{array}{l}58 \\
(14.0)\end{array}$ & $24(11.5)$ & 34 (16.6) & 0.14 \\
\hline \multicolumn{4}{|l|}{ Training level } & 0.60 \\
\hline Med. students & $\begin{array}{l}233 \\
(56.4)\end{array}$ & $120(57.7)$ & $113(55.1)$ & \\
\hline Residents & $\begin{array}{l}180 \\
(43.5)\end{array}$ & $88(42.3)$ & $92(44.8)$ & \\
\hline
\end{tabular}

Residency

program

Emergency $\quad 36$

med. (20.0)

Internal med. $\quad 144$

(80.0)

\begin{tabular}{|c|c|c|c|}
\hline Year of training & & & \\
\hline MS2 & $\begin{array}{l}82 \\
(19.9)\end{array}$ & 41 (19.7) & $41(20.0)$ \\
\hline MS3 & $\begin{array}{l}74 \\
(17.9)\end{array}$ & $40(19.2)$ & 34 (16.6) \\
\hline MS4 & $\begin{array}{l}77 \\
(18.6)\end{array}$ & 39 (18.8) & $38(18.5)$ \\
\hline PGY1 & $\begin{array}{l}64 \\
(15.5)\end{array}$ & $28(13.5)$ & $36(17.6)$ \\
\hline PGY2 & $\begin{array}{l}53 \\
(12.8)\end{array}$ & $27(13.0)$ & $26(12.7)$ \\
\hline PGY3+ & $\begin{array}{l}63 \\
(15.3)\end{array}$ & 33 (15.9) & 30 (14.6) \\
\hline
\end{tabular}
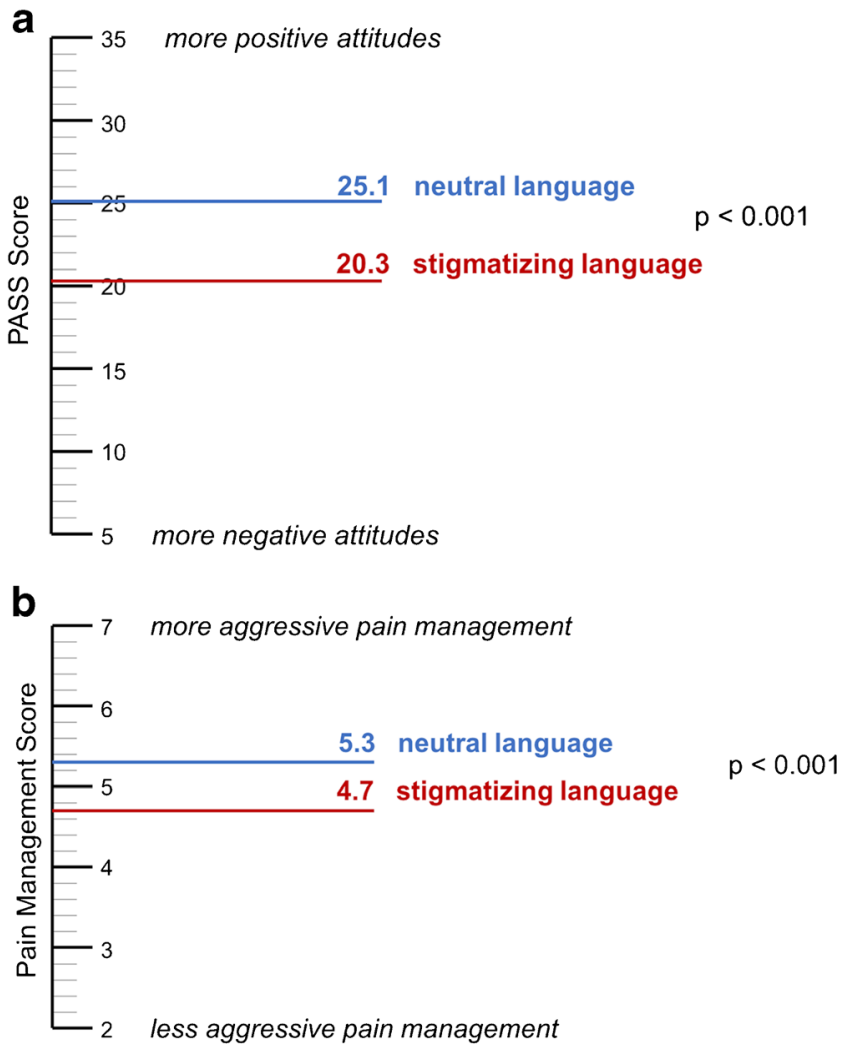

Figure 1 Effect of stigmatizing language on attitudes (Panel A) and on pain management (Panel B).

\section{Additional Predictors of Respondent Attitudes}

Residents' attitudes towards the hypothetical patient were more negative than those of medical students, regardless of study arm (PASS score 20.8 residents vs. 24.2 medical students, $p<0.001)$, and there was an inverse correlation between year of training and PASS score (coefficient $-0.95, p<0.001$ ). Attitudes towards the patient were more positive among respondents who identified as black than among those who did not (24.4 vs. $22.5, p=0.04)$. There was no difference in PASS scores between EM and IM residents.

\section{Comfort in Dosing Pain Medication}

In respondents exposed to the neutral language chart note, comfort in dosing pain medication correlated with more aggressive pain management (coefficient $0.09, p=0.04$ ); however, this correlation was not present in respondents who read the stigmatizing language chart note (coefficient $0.03, p=$ 0.50 ).

\section{Perceptions of Vignette Physician Attitudes}

Respondents who read the stigmatizing language chart note perceived that the physician who wrote the note had more negative attitudes towards the patient, relative to those who read the neutral language chart note (7.6 stigmatizing language vs. 9.2 neutral language, $p<0.001)$. The vast majority $(88 \%)$ of participants who read the neutral language chart note 
thought the physician had neutral or positive attitudes towards the patient (score $\geq 9$ ). Of those who read the stigmatizing language chart note, only $45 \%$ felt the physician had neutral or positive attitudes towards the patient.

Of the approximately 20 differences between the chart notes, almost all were highlighted by respondents who read the stigmatizing language note and perceived that the physician had negative attitudes towards the patient $($ score $<9)$. Frequencies of each linguistic feature that were mentioned by at least five respondents are listed in Table 3. Frequently mentioned phrases among those who read the stigmatizing chart note and perceived a negative attitude were the words "narcotic dependent" and "in our ED frequently," as well as the mention of the girlfriend having shoes on the bed and requesting a bus token. Those who read the neutral note and perceived it to be neutral highlighted the fact that the patient was described as being "in distress" and noted that the girlfriend was by his side.

\section{DISCUSSION}

Language may play a powerful role in influencing clinician attitudes and behavior. Our results suggest that language used in medical records to describe patients can directly influence subsequent physicians-in-training who read the notes, in terms

Table 3 What Did Participants Notice about Each Chart Note? Examples from the Vignettes and Frequency of Mentions by Participants

\begin{tabular}{ll}
\hline \hline Feature of chart note & Stigmatizing chart note \\
& Negative attitude perceived (score <9)* \\
Narcotic dependent & 34 \\
In our ED frequently & 29 \\
Girlfriend on bed with shoes & 24 \\
on & \\
Girlfriends requests bus & 20 \\
token & 16 \\
"Stressful situations" & 15 \\
Use of quotation marks & 14 \\
Cursing at nurse & 10 \\
Insisting pain is "still a 10" & 8 \\
McDonald's & 7 \\
Refuses to wear his oxygen & \\
mask & Neutral chart note \\
& Neutral or positive attitude perceived \\
& $($ score $\geq 9)$ \\
In distress & 26 \\
He believes/potential causes & 23 \\
Describes pain history/ & 17 \\
regimen & 16 \\
Highlighted social factors & 16 \\
Does not question patient's & 16 \\
pain & 12 \\
Wheel himself & 8 \\
Girlfriend is by his side & \\
\hline
\end{tabular}

*The detect score is based on three questions with Likert-type responses, each ranging from 1 to 5, with higher scores indicating that the participant perceived that the admitting physician had more positive attitudes towards the patient. In any question, a score of 3 represents "average." A score $<9$ indicates that the participant selected "less than average" for at least one of the three questions and thus perceived that the admitting physician who wrote the chart note had a somewhat negative attitude towards the patient. of both their attitudes towards the patient and their medicationprescribing behavior. This is an important and overlooked pathway by which bias can be propagated from one clinician to another, further impairing healthcare quality for the individual patient as well as entrenching healthcare disparities overall for those who are stigmatized.

There is growing evidence that the language used to communicate in healthcare reflects and influences clinician attitudes towards their patients. ${ }^{11,23,29,30}$ Finucane and Carrese found that residents were more likely to mention black race (vs. white) when the patient had stereotypically negative behavior (e.g. drug use or treatment nonadherence). ${ }^{29}$ Specific to SCD, Glassberg et al. found that physicians who use the term "sickler" - a term to describe patients with SCD, which is often considered by patients and clinicians to be derogatory - have more negative attitudes towards those patients. ${ }^{30}$ Our study adds to this compelling literature by highlighting the important role of language in the medical record as more than a reflection of attitudes - it is also a means to transmit these attitudes to others.

We found that residents had more negative attitudes than medical students towards the patient. It is notable that attitudes towards patients seem to become more negative as trainees progress; this may be a manifestation of the ethical erosion trainees can experience ${ }^{31}$ as they are influenced by the "hidden curriculum" of observed negative attitudes and behaviors among their peers and seniors in the clinical setting. ${ }^{19-22} \mathrm{We}$ also observed that participants who identified as black or African American had more positive attitudes towards the patient, affirming prior findings that African American clinicians have more positive attitudes towards patients with SCD. ${ }^{13}$

While respondents noted a variety of details that led them to believe that the admitting physician was more or less biased against the patient, a few themes stood out. Some respondents commented on the negative impact of irrelevant comments about behavior and conflicts with the healthcare team, while others noted the positive impact of including psychosocial factors and patient beliefs. A noteworthy finding was the tension in quoting a patient's own words: some respondents felt it was an empathetic practice, while others felt quotes were employed to signal low socioeconomic status or imply factitious histories. The practice of quoting a patient, often encouraged in medical education, ${ }^{32,33}$ deserves a more nuanced analysis of its potential role in providing information and nurturing compassion versus stigmatizing a patient and perpetuating bias. It is remarkable that, when asked to reflect on the note, many respondents were able to identify potentially impactful language - even if earlier they had made their own judgments based on that language. This capacity for reflection may be a promising point of intervention.

One important issue to consider moving forward is what should be documented in the medical record. We have shown that language in medical records may perpetuate bias by negatively impacting the attitudes and decision-making of 
subsequent physicians-in-training. Yet some of the information conveyed in stigmatizing notes-e.g. concerns about a substance use disorder or nonadherence - is important to share with members of the healthcare team. Our study did not aim to address the issue of exactly what should be written, but to understand and consider the consequences. Physicians-in-training may feel both positive and negative emotions about their patients, but value judgments informed by feelings about a patient should not appear in the medical record. Clinicians have a responsibility to record data that are relevant and as objective as possible, as well as to recognize that using certain phrases (e.g. substance abuse vs. substance use) opens the possibility for other clinicians to make or interpret judgment.

This study has several limitations. First, data collection occurred at a single center: a large, urban academic medical center with a significant black patient population. Results may not be generalizable to other healthcare settings. Second, this was a vignette study with a defined set of answer choices to assess attitudes and pain management, so we do not know exactly how these dynamics may manifest in everyday practice. However, the attitudinal scale used was previously validated, and the choices for pain management were made by physicians with expertise in SCD and emergency and inpatient medicine. Importantly, all of the stigmatizing language used to construct the vignette was abstracted from real medical records, so the vignette chart note represents a realistic portrayal of how clinicians may communicate with each other in the medical record. Our chart note had many examples of stigmatizing language; thus, the effect may be stronger than typically seen in a single note.

Finally, the current study did not set out to address which dimensions of the stigmatizing language were the most impactful (e.g. casting doubt using quotation marks vs. blaming the patient for his condition). This was a proof-of-concept study to demonstrate that the language we use in medical records impacts physician-in-training attitudes and decision-making. Future work should evaluate which kinds of stigmatizing language are most likely to perpetuate bias and adversely affect patient care.

Every encounter with a patient is documented in a chart note, including patient symptoms and history, objective signs and results, and the clinician's assessment and plan for the patient. For some patients, the medical record may be the only source of information a new clinician has about them. We must question the assumption that the medical record always represents an objective space. Clinicians must be vigilant to guard against contributing to bias as they write chart notes about their own patients and as they read chart notes written by others. The language in medical records should be more carefully considered to avoid perpetuating clinician biases and the healthcare disparities that may arise from them.
Acknowledgements: This work was supported by the Johns Hopkins University School of Medicine AMWA Dr. Elizabeth Small Grant for Urban Primary Care and the National Heart, Lung, and Blood Institute (RO1HLO88511). We presented an earlier version of the manuscript as an oral presentation at the Johns Hopkins University Medical Student Research Symposium in Baltimore, MD, in February 2017, and as a poster at the Society of General Internal Medicine Annual Meeting in Washington, DC, in April 2017.

Corresponding Author: Anna Goddu, MSc; Johns Hopkins University School of Medicine, Baltimore, MD, USA (e-mail: agoddu1@jhmi.edu).

\section{Compliance with Ethical Standards:}

Conflict of Interest: The authors declare that they do not have a conflict of interest.

Data Statement: The data sets analyzed in this study are available from the corresponding author on reasonable request.

\section{REFERENCES}

1. Nelson A. Unequal treatment: confronting racial and ethnic disparities in health care. J Natl Med Assoc. 2002;94(8):666-8.

2. Drwecki BB, Moore CF, Ward SE, Prkachin KM. Reducing racial disparities in pain treatment: the role of empathy and perspective-taking. Pain. 2011;152(5):1001-1006.

3. Todd KH, Samaroo N, Hoffman JR. Ethnicity as a Risk Factor for Inadequate Emergency Department Analgesia. JAMA. 1993;269(12):1537-1539.

4. Green CR, Anderson KO, Baker TA, et al. The unequal burden of pain: confronting racial and ethnic disparities in pain. Pain Med. 2003;4(3):277-294.

5. Uncapher H, Areán PA. Physicians Are Less Willing to Treat Suicidal Ideation in Older Patients. J Am Geriatr Soc. 2000;48(2):188-192.

6. Reuben DB, Fullerton JT, Tschann JM, Croughan-Minihane M. Attitudes of Beginning Medical Students Toward Older Persons: A FiveCampus Study. J Am Geriatr Soc. 1995;43(12):1430-1436.

7. Kelly PA, Haidet P. Physician overestimation of patient literacy: A potential source of health care disparities. Patient Educ Couns. 2007;66(1): 119-122.

8. Berkman ND, Sheridan SL, Donahue KE, Halpern DJ, Crotty K. Low Health Literacy and Health Outcomes: An Updated Systematic Review. Ann Intern Med. 2011;155(2):97.

9. Forhan M, Salas XR. Inequities in Healthcare: A Review of Bias and Discrimination in Obesity Treatment. Can J Diabetes. 2013;37(3):205-209.

10. MacLean L, Edwards N, Garrard M, Sims-Jones N, Clinton K, Ashley L. Obesity, stigma and public health planning. Health Promot Int. 2009;24(1):88-93.

11. Kelly JF, Westerhoff CM. Does it matter how we refer to individuals with substance-related conditions? A randomized study of two commonly used terms. Int J Drug Policy. 2010;21(3):202-207.

12. Chapman EN, Kaatz A, Carnes M. Physicians and Implicit Bias: How Doctors May Unwittingly Perpetuate Health Care Disparities. J Gen Intern Med. 2013;28(11):1504-1510.

13. Haywood Jr. C, Lanzkron S, Hughes M, Brown R, Saha S, Beach MC. The Association of Clinician Characteristics with their Attitudes Toward Patients with Sickle Cell Disease: Secondary Analyses of a Randomized Controlled Trial. J Natl Med Assoc. 2015;107(2):89-96.

14. Devine PG. Stereotypes and prejudice: their automatic and controlled components. J Pers Soc Psychol. 1989;56(1):5-18.

15. Greenwald AG, Banaji MR, Rudman LA, Farnham SD, Nosek BA, Mellott DS. A unified theory of implicit attitudes, stereotypes, selfesteem, and self-concept. Psychol Rev. 2002;109(1):3-25.

16. Cooper LA, Roter DL, Carson KA et al. The associations of clinicians' implicit attitudes about race with medical visit communication and patient ratings of interpersonal care. Am J Public Health. 2012; 102(5):979-87

17. Green AR, Carney DR, Pallin DJ et al. Implicit bias among physicians and its prediction of thrombolysis decisions for black and white patients. J Gen Intern Med. 2007;22(9):1231-8.

18. Sabin JA, Greenwald AG. The influence of implicit bias on treatment recommendations for 4 common pediatric conditions: pain, urinary tract 
infection, attention deficit hyperactivity disorder, and asthma. Am J Public Health. 2012;102(5):988-95.

19. Hafferty FW, Franks R. The Hidden Curriculum, Ethics Teaching, and the Structure of Medical Education. Acad Med. 1994;69(11):861-871.

20. Burke SE, Dovidio JF, Perry SP et al. Informal Training Experiences and Explicit Bias against African Americans among Medical Students. Soc Pyschol Q. 2017;80(1):65-84.

21. Phelan SM, Burke SE, Hardeman RR et al. Medical School Factors Associated with Changes in Implicit and Explicit Bias Against Gay and Lesbian People among 3494 Graduating Medical Students. J Gen Intern Med. 2017;32(11):1193-1201.

22. Kenison TC, Madu A, Krupat E et al. Through the Veil of Language: Exploring the Hidden Curriculum for the Care of Patients with Limited English Proficiency. Acad Med. 2017;92(1):92-100.

23. Kelly JF, Dow SJ, Westerhoff C. Does Our Choice of Substance-Related Terms Influence Perceptions of Treatment Need? An Empirical Investigation with Two Commonly Used Terms. J Drug Issues. 2010;40(4):805818.

24. Ratanawongsa N, Haywood C, Bediako SM, et al. Health care provider attitudes toward patients with acute vaso-occlusive crisis due to sickle cell disease: Development of a scale. Patient Educ Couns. 2009;76(2):272-278.

25. Alexander CS, Becker HJ. The use of vignettes in survey research. Pub Opin Q. 1978; 42: 93-104.
26. Veloski J, Tai S, Evans AS, Nash DB. Clinical vignette-based surveys: a tool for assessing physician practice variation. Am J Med Qual. 2005; 20: 151-7.

27. O'Conor KJ, Lanzkron S, Haywood C Jr, Beach MC. Implicit Bias and Stigmatizing Language in Medical Records of Patients with Sickle Cell Disease. Society of General Internal Medicine Annual Meeting. Hollywood, FL. 13 May 2016.

28. Link BG and Phelan JC. Conceptualizing Stigma. Annu Rev Sociol. 2001;27:363-85.

29. Finucane TE, Carrese JA. Racial bias in presentation of cases. J Gen Intern Med. 1990;5(2):120-121.

30. Glassberg J, Tanabe P, Richardson L, DeBaun M. Among emergency physicians, use of the term "Sickler" is associated with negative attitudes toward people with sickle cell disease. Am J Hematol. 2013;88(6):532533.

31. Feudtner C, Christakis DA, Christakis NA. Do clinical clerks suffer ethical erosion? Students' perceptions of their ethical environment and personal development. Acad Med. 1994;69(8):670-9.

32. Ball Jw, et al. "Chapter 1: The History and Interviewing Process." From Seidel's Guide to Physical Examination. New York: Elsevier; 2015: 1-20.

33. Bickley LS. "Chapter 1: Overview: Physical Examination and History Taking." In Bates' Guide to Physical Examination and History Taking. Philadelphia: Wolters Kluwer Health; 2013: 3-25. 